\title{
Téoros
}

Revue de recherche en tourisme

\section{Le tourisme en terre amérindienne}

\section{Yves Léger}

Volume 6, numéro 3, décembre 1987

Le tourisme, phénomène mondial

URI : https://id.erudit.org/iderudit/1080606ar

DOI : https://doi.org/10.7202/1080606ar

Aller au sommaire du numéro

Éditeur(s)

Université du Québec à Montréal

ISSN

0712-8657 (imprimé)

1923-2705 (numérique)

Découvrir la revue

Citer cet article

Léger, Y. (1987). Le tourisme en terre amérindienne. Téoros, 6(3), 40-42. https://doi.org/10.7202/1080606ar

Ce document est protégé par la loi sur le droit d'auteur. L'utilisation des services d'Érudit (y compris la reproduction) est assujettie à sa politique d'utilisation que vous pouvez consulter en ligne.

https://apropos.erudit.org/fr/usagers/politique-dutilisation/
Cet article est diffusé et préservé par Érudit.

Érudit est un consortium interuniversitaire sans but lucratif composé de l’Université de Montréal, l'Université Laval et l'Université du Québec à Montréal. Il a pour mission la promotion et la valorisation de la recherche. https://www.erudit.org/fr/ 


\section{Le tourisme en terre

Tourisme lucratif se pliant aux exigences d'une luxueuse clientèle internationale ou élément parmi d'autres d'une politique récréative de plein air? Est-ce ainsi que se pose la question du tourisme en terre amérindienne? Abordant ce thème, on pourra traiter de deux impasses, de trois politiques et d'une multitude d'entreprises possibles.

Mais ce sujet est complexe, controversé et aussi prématuré en ce sens qu'il n'a pas ćté abordé directement par les premiers intêressés. Nous verrons pourquoi?

Le tourisme international est aussi - et toujours - un rapport entre deux cultures. $\mathrm{Si}$, un jour, il se fait vraiment du tourisme en terre amérindienne, il ne pourra être que culturel. Sommes-nous à la veille d'un tel développement? Notre examen de cette question devrait servir d'abord aux discussions sur la nécessité d'une concertation stratégique entre les intervenants du secteur public en tourisme. Face au géant américain, le Canada doit toujours se remettre en question et parfaire ses choix; face au Canada, la société amérindienne est à peu près dans la même situation.

${ }^{7}$ M. Yves Léger a travailié auprés des sociétés amérindiennes après une formation en anthropologle a l'Université Laval: il a commencé de nouvelles études at module de gestion et intervention touristiques de P'UOAM I'an passé.

\section{Le dilemme de l'ouverture aux autres}

Le processus de négociations canadoaméricain sur le libre-échange est l'antithèse de celui des négociations en cours entre les nations amérindiennes et les gouvernements au Canada. Une question fondamentale se pose: dans quelle mesure peut-on affirmer sa différence et en mêtme temps consolider un "partnership" de croissance économique?

Ce dilemme typiquement canadien de pont entre nations distinctes ou entre ćconomies régionales est également le fait des premiers habitants du pays: entre eux, comme par rapport aux autres, ils ont plus que jamais la tâche complexe de se situer sur les plans politiques, culturels et économiques.

Officiellement, une politique globale d'autonomie est poursuivie par les amérindiens. Contrepoids à une situation de dépendance établie, une telle position cherche à instaurer une voie d'émancipation: l'affirmation de soi générant la reconnaissance par l'autre, permettrait la réalisation d'objectifs distinctifs et enfin donner lieu au partenariat...

Le chemin à parcourir est énorme, car le Canada éprouve des réticences à jouer le jeu à l'américaine. Des choix culturels se superposent aux disproportions d'échelle, de rythme et de ressources; comment ne pas comprendre qu'il en est de même, et plus encore, entre la société amérindienne et la société euro-canadienne?

La sensibilité des canadiens, des québécois en particulier, à cette rivalité entre les strictes valeurs économiques et les valeurs culturelles discriminatoires favorise une saine compréhension de la problématique des pays en voie de développement. $\grave{A}$ ce titre, la question amérindienne peut se révéler un miroir des aptitudes du Canada à jouer un rôle de chef de file sur la scène internationale.

Il ne fait pas de doute à mes yeux que la dimension culturelle du tourisme sera de plus en plus l'occasion et le support d'échanges véritables, donc à deux sens, selon l'axe nord-sud. En dépit des problèmes d'infrastructure, de production, de diversification et d'insertion du tiers-monde dans l'économie de marché, la mise en valeur des spécificités et l'échange "librement consenti" entre elles représentent un défi pour l'humanité: cette voie permettrait de rétablir un nouvel équilibre à l'encontre de la tendance peu louable de la concentration du pouvoir et de la richesse.

\section{Les niveaux de nationalismes amérindiens}

La sédentarisation des populations autochtones du Canada est généralement confirmée depuis une génération. La tribu, ou bande, vit désormais en un village qui était par le passé un lieu de rassemblement estival. Les limites territoriales légalement reconnues (les "réserves") ne sont en fait qu'enclaves au sein de l'espace vital traditionnel. L'asservissement de ces larges territoires à la juridiction des gouvernements provinciaux constitue le principal litige à résoudre entre les gouvernements etablis et ceux que les organisations amérindiennes veulent mettre en place.

À la base, la bande équivaut au niveau municipal mais jouit thériquement de l'autonomie politique; ses revendications territoriales s'appuient sur l'occupation passée, les problèmes actuels et les aspirations d'avenir. Responsable de son orientation, elle doit cependant, par la force des choses, déléguer pouvoirs ou représentation à un autre niveau.

La nation regroupe les bandes de même appartenance linguistique dont les territoires originaux sont en principe adjacents. $\AA$ ce niveau, le problème de base prend déjà une proportion considérable. Les nations à leur tour délèguent leurs positions politiques à un niveau supérieur.

L'assemblếe des premières nations est l'organe représentatif de l'ensemble des indiens canadiens inscrits, c'est-à-dire appartenant à une bande (Inuits, Métis et noninscrits ont aussi leurs organisations). Ce nationalisme "dilué" est cependant nécessaire pour transmettre les problèmes fondamentaux au répondant fédéral responsable de la tutelle de fait, de l'asservissement des territoires et droits aboriginaux ainsi que des amendements constitutionnels à produire pour la régularisation des relations entre les deux sociétés.

À la limite, la solidarité amérindienne déborde les frontières canadiennes: analogiquement au concept de "négritude", elle soulève le débat au-delà des préocupations politiques et administratives immédiates... qui du reste sont loin de se résoudre dans un exercice de rondes de négociations, aussi bien conduites soient-elles! 


\section{Tourisme récréatif et tourisme culturel}

Au Québec, le tourisme récréatif est depuis fort longtemps relié aux pratiques de la chasse et de la pêche; le plus récent bilan disponible du M.L.C.P. (1) estime les dépenses engendrées par les activités consommatrices de la faune à $\$ 425$ millions contre $\$ 245$ millions pour les activites non-consommatrices. Ce dernier secteur est d'ailleurs en progression rapide dans la faveur du public québécois. Deux philosophies de plein air $s^{\dagger}$ affrontent: celle de la compétition valorisant la performance par le trophée (ou le panache!) et celle de la participation où détente et communication sont des enjeux suffisants. Un nouveau marche s'instaure donc et on commence à considérer la portée économique d'un tourisme social qui se superpose au tourisme commercial classique $^{(2)}$.

Si la chasse et la pêche sportives sont institutionnalisées dans une gestion complexe (publique, privếe, para-publique), et rationnelle (recherche, quotas de prélèvement, normes d'exploitation, ....), dont le contrôle procure des dividendes politiques et economiques, semble-t-il, un problème se pose dans la reconnaissance des droits et intérêts amérindiens en cette matière. Car dans la culture autochtone, chasse et pêche ne relèvent ni de l'exploit, ni du loisir, ni même du mal nécessaire du contrôle des populations animales! Ces activités sont les assises d'un mode de vie qui soustend une vision du monde: notions d'espace-temps, relations sociales et d'échanges, cycles vitaux, etc. La participation de la famille à la vie en forêt, l'approche du gibier et la disposition de celuici, entre autres, n'ont rien à voir avec la chasse sportive des touristes.

Pourtant, Micmacs, Montagnais, Cris et Attikameks sont impliqués (ou en voie de l'être) dans des entreprises de type "pourvoirie" conventionnelle. Băties en fonction des besoins de la société dominante ou sur pressions de celle-ci (le marché du prélèvement de la faune est bien établi), ces organisations ne rendent cependant pas justice aux aspirations de ces nations qui ont à se faire connaître sous d'autres modes tout aussi bien qu'auprès de nouvelles clientẻles.

Mais un penible constat s'impose: en plus de l'exploitation forestière systêmatique qui a ébranlé l'organisation de plusieur's communautés, les amérindiens déplorent à juste titre que leur patrimoine ait été dếvalorisé, voire méprisét ${ }^{31}$. Ils sont pourtant conscients du fait que les importants investissements dans. les équipements culturels urbains (Expo 67, Québec 1534-1984, ...) sont justifiés en partie par une attractivité plus compétitive sur le marché du tourisme international. Pourquoi alors les limiter à une fonction d'hôtellerie de la forêt ou, pire encore, au rôle de guide qui les dépossède de leurs ressources alimentaires de base?

\section{Un outil de promotion}

Un récent travail nous a confirmé le grand intérèt du marché européen envers un éventuel produit touristique typiquement amérindien $^{(4)}$. Le phénomẻne n'est pas surprenant: une démystification de la grande amérique est en cours dans le vieux continent saturé de lui-même. La cohabitation des gratte-ciel et du chapeau de plume prend un charme romantique, environnementaliste, historique et intellectuel sur l'éternel continuum "nature et culture". Le marché américain lui-même n'est sans doute pas totalement insensible aux particularismes dans la mesure où ils ne salissent pas son propre salon...

Ottawa et Québec ont donc dans ce dossier des cartes valables à jouer. Certes la balance des comptes nationaux bénéficierait d'une revitalisation du tourisme et on ne peut pas dire que le nord ait été réellement mis à contribution jusquà maintenant. De plus, une diminution des paiements de transferts paternalistes aux amérindiens serait la bienvenue. Pour la province, une attitude positive et une intervention bien planifiée entraîneraient des impacts économiques positifs en plus de contribuer à renforcir sa position politique specifique dans l'ensemble canadien. Non pas qu'un "produit" amérindien bien emballe soit à lui seul le remède assuré à l'énorme déficit touristique de la province! Mais du moins il peut être l'occasion d'une révision stratégique en vue d'une relance du tourisme sur l'ensemble du territoire.

La société amérindienne, quant à elle, a tout intérêt à s'élever au rang de partenaire. Il est clair que le concept de "croissance économique", de même que son corollaire de rythme de croissance, se fondent en premier lieu sur une phase de "décollage" qui reste à expérimenter. Sans doute est-elle mal préparée à présenter son plus beau visage, encore moins à impressionner les visiteurs selon des critères qui ne sont pas les siens. Mais en principe, la promotion d'un authentique tourisme culturel aura des effets d'ouverture intellectuelle favorable de part et d'autre. Cet objectif n'est-il pas à l'origine même du tourisme? Et devons-nous nous satisfaire des centres de ski pour contrer l'exode québécois et piquer la curiosité des étrangers pour nos beaux hivers? Et si un court séjour en terre amérindienne êtaît un outil de vente du Palais des congrès?

\section{Projet ponctuel et tourisme intégré}

Il va de soi que l'entreprise privée investirait dans un projet original si les prévisions du marché étaient favorables. Des promoteurs amérindiens commencent à tâter le poul des clientèles et des intervenants possibles. Mais la mise en oeuvre du moindre projet est d'une grande complexité. Il y a deux raisons à cela:

1) Des difficultếs intếrieures risquent de compromettre une initiative $\mathrm{d}^{+}$affaires coupée de ses racines communautaires. Il faut savoir en effet qu'une solidarité fonctionnelle existe depuis toujours entre les groupes de production (les familles élargies) de même qu'entre les ressources disponibles (le gibier et les parcelles de territoires). Interdépendants, ni les uns ni les autres ne sont fixes, divisibles ou autrement asservissables. Une intervention d'entreprise doit donc au préalable rencontrer un consensus qui ne peut ètre dicté par des étrangers. Les modalitếs et les contraintes de ce cheminement sont difficilement compréhensibles aux gens de l'extérieur.

2) À cause du manque de ressources financières, les opérations commerciales envisagées jusqu'à maintenant sont sujettes aux normes administratives des sources de financement relevant des autorités fédérales ainsi qu'aux règles d'exploitation ou de statut où l'administration provinciale brouille les perspectives. Car si les premières peuvent à la rigueur s'avérer acceptables, la juridiction en matière d'exploitation territoriale est l'objet d'un litige essentiel.

C'est pourquoi le tourisme en terre amérindienne ne peut actuellement, ni en pratique ni en théorie, être énoncé comme une priorité malgré le potentiel de développement qu'il présente. Au plus, certains compromis sont consentis de part et d'autre pour réduire des tensions ou ouvrir des portes en temporisant sur le fond de la question. Et l'improvisation se poursuit! Le cas des Cris de la Baie James est évidemment exceptionnel puisqu'une entente globale a été ratifiée en bonne et due forme.

Dans ce contexte, ni l'une ni l'autre des sociétés ne s'est engagée dans la voie d'une politique intégrée du tourisme. Car si les institutions amérindiennes sont jeunes et leurs efforts de planification embryonnaires, que dire de notre politique et de notre systeme administratif concernant le tourisme? Quelle cohésion existe-t-il entre Tourisme Canada, l'expansion régionale, les affaires indiennes au fédéral et au provincial, le loisir, chasse et pêche, énergie et ressources, le tourisme et les affaires culturelles pour ne mentionner que ces intervenants?

Jacques Demers a d'abord soulevé la question: "Pierre Lainé insiste, avec raison d'ailleurs, sur la nécessité d'une planification harmonisée du développement (...) n'est-il pas absurde qu'une activite aussi cruciale que le tourisme soit balloté par des mentalités compartimentées, sectorielles, à la merci d'intérêts souvent particuliers? (...) C'est l'avenir des communautés de base qui est en jeu, de même que le caractère du tourisme futur ${ }^{*}(5)$

Un tel constat critique appelle la discussion et les énergies sur un thème d'intérêt commun, le tourisme culturel, qui pourrait s'avérer fort constructif à l'avenir tant sur les plans intérieurs qu'extérieurs. "Il s'agit de l'appli- 
cation concrète et planifiec, tant dans l'espace que dans le temps, de principes énoncés dans la déclaration québécoise sur le tourisme culturel en $1979^{\circ,(6)}$.

On note cependant une volonté récente mais ferme de concertation des autorités gouvernementales et une ouverture sur le potentiel touristique de l'élément culturel et d'un éventuel produit touristique autochtone.

La réunion fédérale-provinciale-territoriale des ministres du tourisme (juin 1987) a identifié la problématique générale et pris position sur les principales lacunes à combler. Retenons pour notre propos le passage suivant: "'L'étude Longwood a révélé l'importance considérable de notre particularité culturelle lorsqu'il s'agit d'attirer les visiteurs américains et, probablement, les visiteurs européens et japonais. Étant donné les constatations de cette êtude, Tourisme Canada a choisi le produit touristique autochtone comme nouvel élément à développer. $(. . .)^{(7)}$

\section{Une vision à long terme}

À Québec dernièrement ${ }^{(8)}$, se tenait un colloque sous le thème: "Tourisme culturel découvrir le génie du lieu". Quelques points essentiels, développés par les conférenciers invités, résument la problématique générale du tourisme en terre amérindienne.

Par opposition à l'industrie soucieuse d'occuper le temps des touristes par une intendance bien rodée, Marc Laplante insiste sur la dimension immatérielle, symbolique du produit touristique recherché par le visiteur: le "frisson" de pénćtrer tant soit peu un autre univers culturel serait l'élément à préserver pour garantir la pérennité d'un produit alors que la banalisation a provoqué une tendance généralisée à rechercher toujours la nouveauté. Les gens se tournent vers un extérieur facilement renouvelable faute d'une profondeur résonnante à leur propre recherche intérieure. Le tourisme serait-il uniquement un mouvement de fuite?

Face à la superficialité de l'offre et à la consommation de masse générées par l'exploitation d'attraits majeurs. Paul-Louis Martin évoque la participation du visité, la mise en valeur du "tissu de base" et les segments de clientèles sensibles à des thèmes originaux où la compréhension du milieu d'accueil est plus satisfaisante que la consommation de clichés à vérifier.

On concoit qu'une telle vision s'accorde mal avec celle du rendement attendu à court terme sur un investissement. La voie du développement économique doit-elle nécessairement passer par le nivellement des valeurs culturelles et l'application des recettes utilisées jusqu'à maintenant par l'industrie du voyage?

Nous sommes tentés d'admettre que le choix de société qui se trame chez nos frères amé- rindiens est le prolongement d'une réflexion de fond toujours non résolue pour nousmêmes: à peine une génération depuis la révolution tranquille et voilà qu'un message de non-urgence nous provient de ceux-là même qui, selon toute apparence, devraient mettre les bouchées doubles dans l'ajustement au système économique libéral.

L'opportunisme va de soi en affaires: la rationnalité économique suppose une vitesse de réaction à toute modifications de la conjoncture. Sans doute les Cris, obligés à la négociation en 1971, ont-ils fait preuve de cette capacité; leur sort n'en demeure pas moins lié à l'ensemble des nations autochtones du Canada. De la même façon, chacune des régions du Québec a sa propre dynamique à établir tout en resserrant des liens d'interdépendance dans la définition d'un produit touristique distinctif, celui du Québec.

N'est-ce pas précisément le rôle de l'état de préserver l'équilibre entre les intérêts communs et les intérêts particuliers des citoyens? Le nivellement des personnalités qui le composent ne serait-il pas, à long terme, une perte qualitative importante du patrimoine exploitable? Nous voici revenus à la difference fondamentale, qui persiste toujours (!), entre les politiques canadiennes et américaines...

\section{Conclusion}

Le phénomène touristique correspond à un processus d'ouverture qui se manifeste au niveau des individus, des organisations et enfin des institutions. L'amélioration généralisée des communications, de l'éducation et du revenu disponible dans le pays industrialisés suscitent l'intérết tout en permettant un mouvement croissant d'échanges physiques et intellectuels sur la planète. Comme le développement de la technologie: une société doit en tenir compte, faire des choix et ajuster son rythme.

Le dilemme du développement de la société amérindienne est analogue à celui du Canada face à la libéralisation des échanges chère au président Reagan. Combien de canadiens la souhaitent vraiment? N'y a-t-il pas entre les individus tout comme entre les pays ou nations des différences essentielles à admettre, donc à affirmer? Par contre, l'évolution de la vie n' impose-t-elle pas l'ajustement de toute entité à son environnement?

Il est indubitable que le Canada, pour de multiples raisons mais en particulier par sa position de médiateur sur le plan international, est le point de mire de nombreux pays en voie de développement. Le problème des minorités nationales pose des contraintes et notre société, de ce point de vue, s'est donné de sérieuses responsabilités. Il y a un prix à payer, mais des avantages à en retirer. Le tourisme culturel avec les amérindiens comme partenaires: une laboricuse négociation qui est un investissement dans l'avenir!

\section{Notes}

(1) DUCHARME, J.L. et PELLETIER, J, Le loisir relie à I'utilisation de la faune au Québec. Études auprès de la population, M.L.C.P., Direction générale de la faune, (non daté)

(2) BODSON, P., STAFFORD, J., Elements pour une économie du tourisme social, in Téoros, vol. 1, no 3. UQAM, 1982.

(3) CONSEIL ATTIKAMEK-MONTAGNAIS, Rapport: sur l'occupation et l'utilisation des territoires par les Attikameks et les Montagnais, 1983, (non publié).

(4) ChAREST, D., LEGER, Y., OUVRARD, S., Plan de marketing d'une pourvoierie amérindienne, Module de G.I.T., UQAM, 1987 (non publié).

(5) DEMERS, J_, Lê tourisme en péril, Ed. Nouwelle Optique, 1983, pp. 194-195.

(6) MAYRAND, P. La culture offerte en partage: un miroir à aloweftes/, in T6oros, vol. 2, no 2, UQAM, 1983.

(7) REUNION FEDERALE-PROVINCIALE-TERRITO RIALE DES MINISTRES DU TOURISME, Document de travail au sujet d'un eadre pour le dóveloppement du produit touristique au Canada, Québec, 1 et 2 juin 1987 lannexe $C_{\text {- }}$ braluation du produit, $\mathrm{p}$. 13 .

(8) FONDATION CANADIENNE POUR LA PROTEC TION DU PATRIMOINE, 14e Confürence: Séminaire de Quebec, 24-27 septembre 1987. 